\title{
ANALYTICAL STUDY AND TRACKING SIMULATIONS OF THE BEAM-BEAM COMPENSATION AT TEVATRON
}

\author{
D. Shatilov, BINP, Novosibirsk, Russia \\ Y. Alexahin, V. Shiltsev, FNAL, Batavia, IL 60510, USA
}

\begin{abstract}
Head-on and long-range induced tunespread of about 0.025 in the Tevatron collider at Run II (together with the increased strength of the resonances) can significantly deteriorate the $\bar{p}$ lifetime and the collider luminosity. It was proposed to employ the so-called Tevatron Electron Lenses (TEL) to compress the beam-beam footprint and eliminate completely the bunch-to-bunch tunespread (PACMAN effect) for small amplitude particles. The first lens has been recently installed and tested [1]. This report presents results of analytical studies and tracking simulations of the linear beam-beam compensation (elimination of the bunchto-bunch tune variation). Compression of the beam-beam footprint (nonlinear compensation) is discussed in [2].
\end{abstract}

\section{INTRODUCTION}

During RUN II with 36 bunches in each beam, the bunchto-bunch tune spread will be about $\delta \nu \approx 0.007$, while the single bunch tune spread will be about $\Delta \nu \approx 0.018$ (see Fig.1). The main problem occurs with the first and the last bunches in the 3 identical trains of 12 bunches each. Tunes of bunches \#1 and \#12 are shifted down and left, respectively, compared to all other bunches, that results in their lifetime deterioration (so-called PACMAN effect). An electron lens, consisting of low energy, high current electron beam colliding with antiprotons, can induce a tuneshift of the antiproton bunches. The electron beam current is modulated, so each antiproton bunch collides with the different electron beam density. With appropriate choice of parameters two such lenses could provide effective compensation of the bunch-to-bunch tunespread.

\section{NUMERICAL SIMULATIONS}

\subsection{Simulation Goals}

The main goal of our simulations is to demonstrate the possibility of using TEL for eliminating the bunch-to-bunch tune spread. We studied how the TEL affects the nonlinear beam dynamics and what general conditions ( $e$-beam radius and profile, misalignment, stability, etc.) must be satisfied in order to keep $\bar{p}$-beam stable. We assume here that these conditions should be more or less general, that is do not depend very much on the working point (if it is chosen properly, of course).

We started with a small betatron tune scan around some working point. The tune variations were about the bunchto-bunch spread, and the goal was to find some "good" and

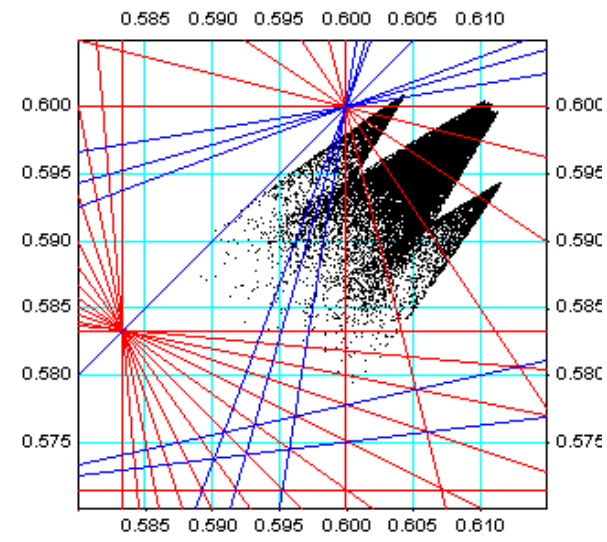

Figure 1: Tunes for the particles with the different betatron amplitudes, for different bunches in the train. The bare lattice tunes are $(20.585,20.575)$. Resonances up to 12 th order are shown.

"bad" working points within this area. Then, we applied the TEL in the "bad" working point in order to shift it to the "good" one and to see the positive effect. After that we varied different TEL parameters and applied some perturbations to see how they affect the resulting $\bar{p}$ distribution. Comparison with the natural "good" working point give us important information how the TEL itself introduces perturbations (linear and non-linear) to the $\bar{p}$-beam.

\subsection{Choice of the Working Point}

As the analytical study [3] shows, the standard Tevatron working point $(20.586,20.576)$ is not a good one due to the proximity of 5 th and 12th order resonances (see Fig.1), and we decided to look at the vicinity of the point $(20.566,20.556)$ surrounded by resonances of higher order (7th). Then, we tried to find such a pair of the "bad" and "good" points that the "bad" one could be shifted to the "good" with only one TEL of two, to simplify the further studies. We found, after a small tune scan, the pair of $(20.566,20.556)$ and $(20.556,20.546)$ points, which requires only TEL2 located at F0 (as planned initially), where the beta-functions are approximately equal. Now the other location is chosen for TEL2, with $\beta_{x} \ll \beta_{y}$, while $\beta_{x} \gg \beta_{y}$ at TEL1. Besides the better operation conditions for the tune shifts, using two TELs with unequal beta-functions strongly reduces excitation of coupling resonances by TELs themselves [3], so the present results can be regarded as a conservative estimate of the effect. 
It should be noticed that some parameters, such as amplitudes of the noise, were intentionally chosen large in order to obtain visible effects of $\bar{p}$-bunch degradation during limited simulation time ( 1 minute of the Tevatron real time). However, we believe that proper scaling of the obtained emittance growth rates and particle loss rates can give more or less realistic estimates of the most important effects relevant to the interaction between the TEL and $p, \bar{p}$-beams.

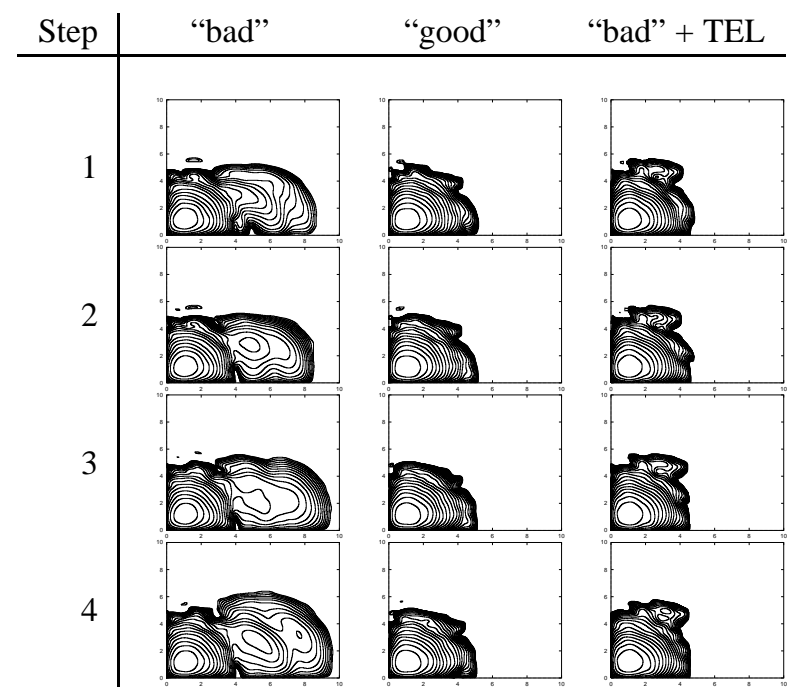

Figure 2: Distribution of the $\bar{p}$-beam in the plane of normalized betatron amplitudes after $(1,2,3,4) \times 3 \cdot 10^{5}$ turns.

An example of simulation results is presented in Fig. 2, where the contour plots of $\bar{p}$ distribution in $\left(A_{x}, A_{y}\right)$ plane are shown. The distance between successive contour lines is $\sqrt{e}$. Each step corresponds to $3 \cdot 10^{5}$ turns, 3000 particles were tracked. The data gathered are averaged over all the particles, all the turns, that is $9 \cdot 10^{8}$ particle-turns for each step. The last column presents the effect of TEL, which is applied in the "bad" working point and shifts it toward the "good" one. We chose here very big radius of the electron beam $\left(3 \mathrm{~mm} \approx 6 \sigma_{\bar{p}}\right)$ just to see the positive effect. The allowable $e$-beam sizes, as well as other parameters, will be discussed in the next sections.

\subsection{Electron Beam Size and Profile}

Since the TEL length $L \ll \beta$, transformation through the electron beam can be presented as a single transverse kick depending on the $\bar{p}$-particle coordinates. The kick consists of two parts: electric and magnetic ones. The first one depends on the $e$-beam distribution density, while the second depends also on the distribution velocity which is not constant due to space charge effects. For tracking purposes, it is convenient to define some function $\rho(r)$ so that the radial kick is equal to:

$$
\delta \dot{r}=\frac{\int_{0}^{r} \rho(t) \cdot 2 \pi t d t}{r}
$$

Note that $\rho(r)$ is not a pure distribution density, since it contains also the contribution of the magnetic force. A linear lens corresponds to $\rho(r)=$ const for $r \leq R_{o}$. The $e$-beam radius $R_{o}$ must be large enough that all the particles in $\bar{p}$ beam feel the same tune shift, but it is limited by the achievable $e$-beam current which is proportional to $R_{o}^{2}$. The real electron beam profile is more complicated and "smoothed", it can also be controlled by a special electrode near the cathode [4].

We tried different $e$-beam profiles and first of all varied the $e$-beam radius to find the minimum acceptable value. Fig. 3 shows the relative luminosity drop after $3 \cdot 10^{6}$ turns w.r.t. initial luminosity versus total electron current for different distributions. One can see that smoother the distribution, the less luminosity drop we observe (i.e., the Gaussian one is the best of three), but in general the total peak electron current required to keep the luminosity is approximately the same for all three distribution functions - about 7A (that corresponds to $R_{o} \approx 3 \sigma_{\bar{p}}$ ) to shift the tune by -0.01 .

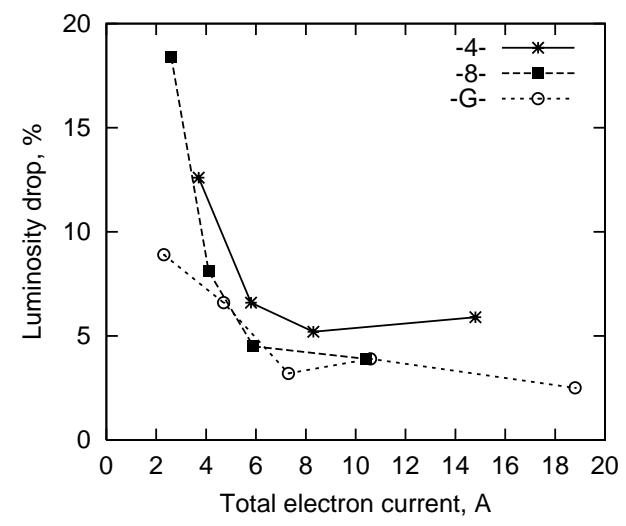

Figure 3: Luminosity drop versus $e$-beam current for the different profiles. The line marked " 4 " corresponds to $\rho(r)=\rho_{o} /\left(1+\left(r / R_{o}\right)^{4}\right)$, line marked " 8 " corresponds to $\rho(r)=\rho_{o} /\left(1+\left(r / R_{o}\right)^{8}\right)$, line marked " $\mathrm{G}$ " corresponds to $\rho(r)=\rho_{o} \cdot \exp \left(-r^{2} / 2 R_{o}^{2}\right)$.

As for $\bar{p}$-beam tails and lifetime, they can be somehow controlled by small modifications of the distribution function $\rho(r)$. The simulations showed that the effect is very sensitive to the $e$-beam profile (and, obviously, to the working point) and further optimization should be performed. It is important to note that the nonlinear compensation (see [2]), with the proper choice of the working point, may give better results with even smaller $e$-beam radius! The obtained value of 7A corresponds to the case of linear compensation without nonlinear optimization and can be considered as an upper limit required to keep the $\bar{p}$-beam stable.

\subsection{Straightness of the Electron Beam}

The electron beam trajectory in the TEL follows strong solenoidal magnetic field lines, and any magnetic error re- 
sults in $e$-beam trajectory distortions. Since the effect of the TEL can be reduced to a single transverse kick, any changes in $e$-beam trajectory are equivalent to some modifications of the distribution function. In our simulations we tried a "curved" $e$-beam with the following horizontal offset between electron and antiproton beams:

$$
d_{x}(z)=d_{o} \cdot \cos (\pi(2 z / L-1))
$$

where $z$ is longitudinal coordinate in the range of 0 to $L$ (solenoid length), $d_{o}=0.5 \mathrm{~mm}$. Measured magnetic field quality is found to be even better [5]. Numerical simulations confirmed that it is acceptable and does not worsen $\bar{p}$-beam dynamics (losses and emittances). Moreover, it is worth to mention that variation of the $e$-beam trajectory by additional orbit correctors (potentially, as many as 20) opens a lot possibilities to control effective integral transverse distribution $\rho(x, y)$. That is especially promising for the future nonlinear compensation, but even for linear BBC it can help to adjust $e$-beam profile in order to shorten the $\bar{p}$ beam tails.

\subsection{Misalignment of the Electron and Antipro- ton Beams}

Misalignment of the $e$ - and $\bar{p}$-beams breaks the symmetry of the beam-beam kick. The most dangerous effects are expected when the edge of electron lens overlaps with the antiproton beam. Simulations showed that misalignment of $d_{x}=0.4 \mathrm{~mm}$ is acceptable for $R_{o}=2 \mathrm{~mm}$ : we observed small changes in the tails, but the luminosity, as well as $\bar{p}$ beam lifetime, was not affected. However, for smaller $e$ beam radius more accurate alignment is required. In any case, the design of the TEL magnetic system includes a number of dipole correctors, so that the electron trajectory can be corrected to coincide, with the required accuracy, with the antiproton orbit in the device.

Nevertheless, the process of bringing two beams into collision can produce damage to the $\bar{p}$-beam. We studied how the resulting distribution depends on the speed of this process. An initial misalignment was chosen to be $d_{x}=5 \mathrm{~mm}$ and decreased exponentially with the time constants $\tau=30$ and $6 \mathrm{sec}$, the $e$-beam radius was $R_{o}=2 \mathrm{~mm}$. The main perturbations $\bar{p}$-beam experiences when overlapping with the edge of electron beam, that corresponds to the shift between beams' centers $d_{x}=1.5 \div 2.5 \mathrm{~mm}$. Just at that time we observed the emittance growth and luminosity decrease, after that the beam core remains more or less stable. Thus we conclude that alignment of the electron beam, if started with large initial shifts (1-2 mm or more), destroys the antiproton beam. The solution is to perform this operation in few steps, each time injecting new $\bar{p}$-beam, or use smaller electron beam current for initial alignment.

\subsection{Effects of the Electron Beam Noises}

Random fluctuations of the electron current from turn to turn, as well as transverse motion of the electron beam, cause antiproton emittance growth. Analytical studies of the issue [6], [7] give the emittance evolution equations. The emittance grows exponentially due to electron current fluctuations:

$$
\varepsilon_{z}(t) \approx \varepsilon_{z o} \cdot \exp \left(8 \pi^{2} t f_{o}\left(\xi_{z 1}^{2}+\xi_{z 2}^{2}\right)\left(\frac{\delta J}{J}\right)^{2}\right)
$$

where $z$ stands for $x$ or $y, \xi_{z 1,2}$ are the values of tune shifts produced by two electron lenses, $\delta J / J$ is the rms value of relative current fluctuations. Transverse motion of the $e$ beam results in linear emittance growth:

$$
\frac{d \varepsilon_{z}}{d t}=8 \pi^{2} f_{o}\left(\frac{\xi_{z 1}^{2}}{\beta_{z 1}}+\frac{\xi_{z 2}^{2}}{\beta_{z 2}}\right) \cdot(\delta Z)^{2}
$$

where $\delta Z$ is the rms electron beam vibration amplitude.

Our numerical simulations are in good agreement with these equations, which set strong requirements on the electron beam stability (see, estimates in [8]). So, we got an emittance growth by a factor of 2.2 during 1 min of the beam time with $\delta J / J=5 \cdot 10^{-3}$, while (3) gives the factor of 1.8 . Note that there are also another sources of emittance growth which are taken into account in the simulations and not included in (3).

\section{CONCLUSION}

We performed analitical study and tracking simulations of the electron lens in Tevatron, which helped us to realize the requirements and to choose the TEL parameters. The first experimental results demonstrate the feasibility and operation of the electron lens. The first TEL has been installed in the Tevatron, commissioned and demonstrated theoretically predicted shift of betatron frequencies of high energy proton beam due to high current low energy electron beam [1]. Tuneshifts of $980 \mathrm{GeV}$ protons of about $\mathrm{dQ}=+0.007$ were achieved with some $3 \mathrm{~A}$ of the electron beam current while the proton lifetime was in the range of 10 to 24 hours. The experimental observations so far are in good agreement with analytical and simulation results (see [1]). In particular, strong dependence of the $\bar{p}$ lifetime on electron beam alignment is reported.

We would like to thank the PC Farms Group in FNAL Computer Division for the opportunity of using farms for our simulations.

\section{REFERENCES}

[1] V. Shiltsev, et al., these Proceedings.

[2] Y. Alexahin, et al., these Proceedings.

[3] Y. Alexahin, FERMILAB-TM-2148, 2001.

[4] N. Solyak, et al., these Proceedings.

[5] K. Bishofberger, et al., these Proceedings.

[6] G. Stupakov, Report No. SSSL-575 (1992).

[7] V. Lebedev, et al., Part. Acc. 44 (1994) 147.

[8] V. Shiltsev, et al., Phys. Rev. ST-AB, 2, 071001 (1999).

[9] D. Shatilov, V. Shiltsev, FERMILAB-TM-2124, 2000. 